\title{
ENVOLVIMENTO DE MATERIAIS SUSTENTÁVEIS EM TEMAS DE TRABALHOS DE CONCLUSÃO DE CURSO DA FACULDADE DE ENGENHARIA MECÂNICA DA UFPA
}

DOI: 10.37702/2175-957X.COBENGE.2021.3444

Léo César de Oliveira Pereira - leocesaroliveira@hotmail.com

Universidade Federal do Pará

Conjunto Guajará I Tv WE 63A 1421

67143-390 - Ananindeua - PA

Roberto Tetsuo Fujiyama - fujiyama@ufpa.br

Universidade Federal do Pará

AV. TAVARES BASTOS 438/203

66613-005 - BELEM - PA

Resumo: $O$ uso de materiais ecologicamente corretos e a sustentabilidade socioambiental são premissas para adequar a tecnologia, o bem estar social à preservação ambiental. Diante disto, o presente trabalho apresenta de forma quantitativa o uso da sustentabilidade em temáticas de Trabalhos de Conclusão de Curso (TCC) da Faculdade de Engenharia Mecânica da Universidade Federal do Pará (UFPA) entre os anos de 2010 e 2020 de forma a evidenciar a importância deste tema na formação do profissional em engenharia. Para isto foi utilizado como banco de dados o currículo Lattes dos professores que compõem o corpo docente da Faculdade de Engenharia Mecânica (FEM) da UFPA em busca das orientações de TCC's concluídas. Os resultados mostram o avanço na elaboração de TCC ao longo do tempo como pretende o presente artigo. Os resultados também deixam bem definidos que as áreas da engenharia mecânica que aprestaram maior quantidade de TCC's foram as de Térmicas e Fluidos e de Materiais e Processos de Fabricação.

Palavras-chave: Sustentabilidade. Trabalho de conclusão de curso. Engenharia. Educação. 


\section{INTRODUÇÃO}

A sustentabilidade socioambiental é um desafio diante dos vários aspectos imbricados que envolvem a mesma e a produção de ciência e tecnologia. $O$ desenvolvimento científico e tecnológico acelerado desencadeia mudanças sociais que promovem demandas por tecnologias para a melhoria da qualidade de vida material e de bem estar das pessoas acarretando a busca por formas de controle da tecnologia (VESRASZTO et al, 2009).

Diante disto, a educação de nível superior tem um papel prioritário à medida que os futuros profissionais são os que trabalharão com os recursos sociais, ambientais e econômicos e, portanto, precisam perceber sua função na busca por transformações sociais e melhoria de bem-estar para as pessoas das gerações atuais e das próximas (LOUREIRO, PEREIRA \& PACHECO JR., 2016). Por isso, a universidade tem sido reconhecida como elemento-chave na compreensão das questões ambientais e sociais complexas derivadas da implementação da sustentabilidade (BARTH; RIECKMANN, 2012; WRIGHT; HORST, 2013).

No Brasil, alguns pesquisadores, dentre os quais, Bazzo, Pereira e Linsingen (2000), Linsingen (2002), Linsingen, Pereira e Bazzo (2003), Brito (2011), Carletto (2011), Dwek, Coutinho e Matheus (2011), há algum tempo, apontam a necessidade de se redirecionar a formação tecnológica da área de engenharia na perspectiva de que os futuros engenheiros(as) percebam as múltiplas implicações da engenharia com as dimensões sociais, ecológicas, culturais e econômicas da tecnologia.

Segundo Vieira \& Coelho (2017), a região norte detém de 4\% das vagas brasileiras nos cursos de engenharia, tem o estado do Amazonas como o maior detentor de vagas nos cursos de Engenharias com $48 \%$ das vagas (12.878 vagas), seguido pelo o estado do Pará com $25 \%$, Tocantins com 10\%, Amapá com $9 \%$, Rondônia com $5 \%$, Acre com $2 \%$ e por ultimo o Estado do Roraima com apenas 1\%.

Nesses cursos de engenharias é estudado de forma geral o desenvolvimento de novos materiais, aplicação de materiais e caracterização de materiais, onde as pesquisas visando materiais com menor impacto ambiental são tratadas com atenção.

A Universidade Federal do Pará vincula seus cursos a departamentos e institutos e o instituto ao qual é vinculada a Faculdade de Engenharia Mecânica (FEM) é o Instituto de Tecnologia (ITEC). O instituto de Tecnologia da UFPA conta ainda com 08 Faculdades de Engenharias além da Engenharia Mecânica. Na Figura 1 tem-se ilustrado a fachada frontal do Instituto de Tecnologia da UFPA, que devido a pandemia encontra-se em isolamento por questão de segurança. 
Figura 1 - Fachada do Instituto de Tecnologia da

Universidade Federal do Pará onde fica localizada a

Faculdade de Engenharia Mecânica.

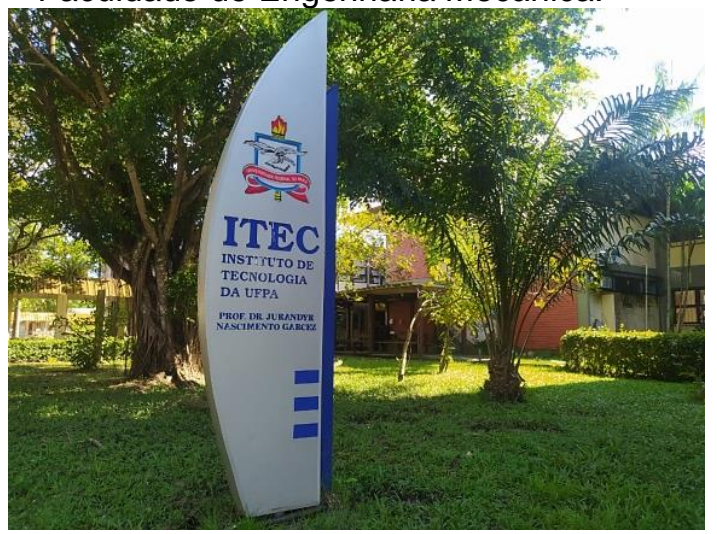

Fonte: Autoria própria (2021).

O curso de engenharia mecânica conta também com o Laboratório de Engenharia Mecânica (LABEM), onde se estrutura a maior parte das atividades dos grupos de pesquisas. O quadro de corpo docente da Faculdade de Engenharia Mecânica da UFPA conta com 30 professores efetivos que se distribuem em quatro áreas de concentração: Térmicas e fluidos, Materiais e processos de fabricação, Sistemas mecânicos e Gestão e Manutenção. O Gráfico ilustra a alocação do quantitativo de professores por área de concentração. A Figura 2 ilustra o (LABEM), devido à pandemia as atividades laboratoriais e administrativas também estão restritas à extrema necessidade.

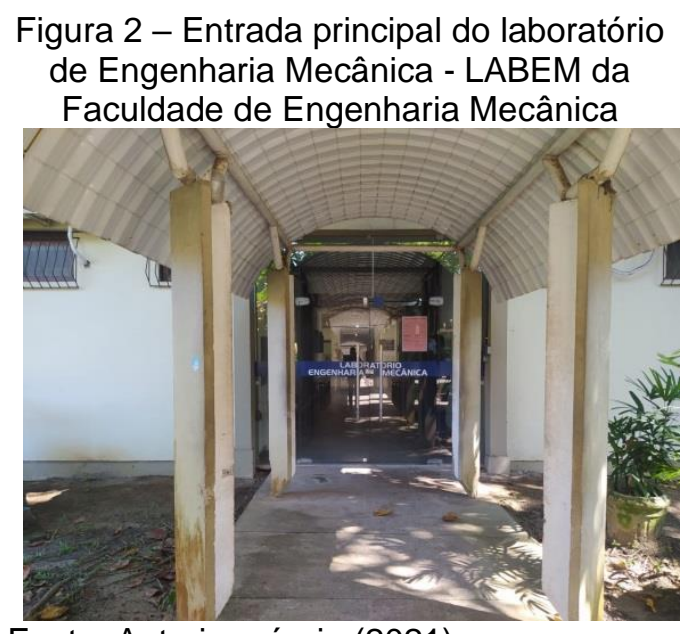

Fonte: Autoria própria (2021).

A Faculdade de Engenharia Mecânica atualmente conta com 30 professores permanentes e as áreas de trabalhos são térmicas e fluidos, materiais e processos de fabricação, sistemas mecânicos e gestão e manutenção. Atualmente conta com 10, 09, 08 e 03 professores respectivamente em cada uma das áreas citadas.

O Curso de Engenharia Mecânica é responsável pela formatura de Engenheiros semestralmente e além de direcionar os graduados para o mercado de trabalho, também é responsável pela alimentação de mestrando para o Programa de pós-Graduação em Engenharia Mecânica - PPGEM. Sendo que o PPGEM tem grande em suas áreas de pesquisa linhas de pesquisa com foco em sustentabilidade. 
Neste artigo pretende-se abordar uma análise qualitativa e quantitativa de Trabalho de Conclusão de Curso que foram produzidos no intervalo entre 2010 e 2020 e que conduziram a formatura de Engenheiros Mecânicos.

\section{METODOLOGIA}

\subsection{Caracterização da pesquisa}

Esta pesquisa do ponto de vista de abordagem se caracteriza como quantitativa. No caso este estudo apresenta de forma sucinta a presença da sustentabilidade como temática em Trabalhos de Conclusão de Curso de Engenharia Mecânica da Universidade Federal do Pará, quantificando as pesquisas relacionadas a isto. $O$ trabalho visa mostrar a importância de este assunto ser discutido em fase de formação profissional do estudante e como é essencial que a dinâmica empregada nos grupos de pesquisas direcione seus trabalhos a esta temática.

\subsection{Coleta de dados}

Utilizou-se os dados da Faculdade de Engenharia Mecânica (FEM) para a busca do corpo docente do curso. Após isto foi realizada uma análise curricular, na plataforma Lattes, verificando a existência de orientações concluídas de trabalhos de conclusão de curso com temáticas relevantes abrangendo a Sustentabilidade. Foi estabelecido o período entre 2010 até 2020 para identificação desta temática

Como critério de inclusão foi estabelecido que temáticas que tratassem de fontes renováveis de energia, materiais de origem renovável, reaproveitamento de rejeitos, entre outros assuntos pertinentes a sustentabilidade socioambiental nas mais diversas áreas de abrangência da Engenharia Mecânica.

Os dados foram separados e avaliados de acordo com as áreas de concentração do curso de engenharia mecânica da Faculdade de Engenharia Mecânica.

\section{$3 \quad$ RESULTADOS E DISCUSSÕES}

De posse do banco de dados obtidos conforme descrito no item metodologia as informações foram tratadas de forma a avaliar o contexto do tema proposto nesta pesquisa e artigo.

O gráfico da Figura 3 representa a quantidade de Trabalhos de Conclusão de Curso que envolvem a sustentabilidade por área de concentração de estudo, segundo o levantamento efetuado por orientador e ao longo dos anos entre 2010 e 2020. 
Figura 3 - Quantidade de TCC's defendidos no intervalo de 2010 a 2020 na Faculdade de Engenharia Mecânica.

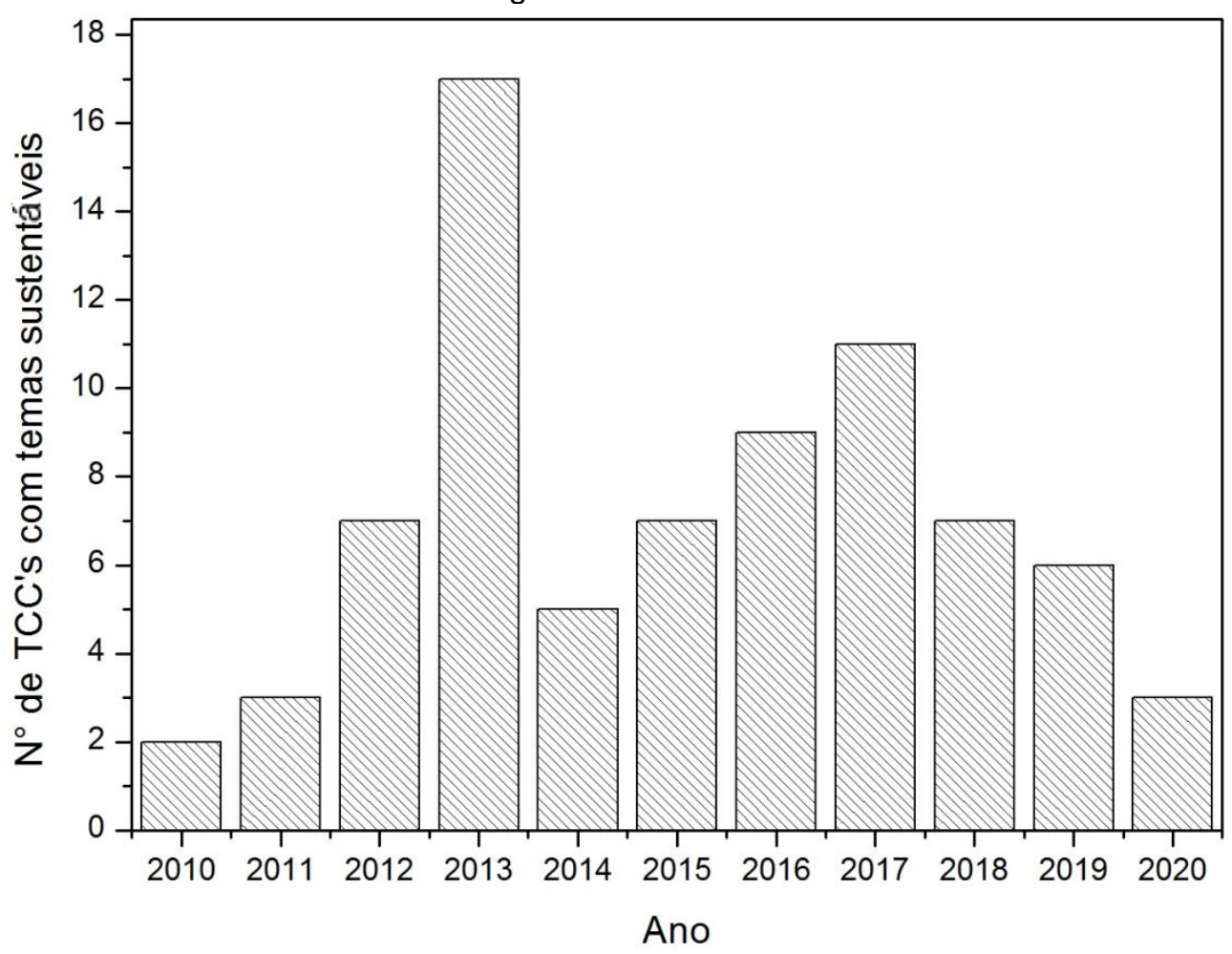

Fonte: Autoria própria (2021).

Fazendo-se uma análise mais particular, pode-se observar que dentre os anos de 2010 e 2020, foram nos anos de 2013 e 2017 que a sustentabilidade se destacou nos trabalhos de conclusão de curso, totalizando $22,07 \%$ e $14,28 \%$, respectivamente, do total de trabalhos. No ano de 2020 devido a questões pandêmicas a Universidade Federal do Pará fechou as portas por um grande período de tempo o que pode ter acarretado a dificuldade na orientação e no andamento dos trabalhos acadêmicos. Ainda observando $\mathrm{O}$ gráfico da Figura 3 notou-se que houve um acentuado crescimento em pesquisas relacionadas à sustentabilidade entre os anos de 2010 e 2013.

No total foram encontrados 77 Trabalhos de Conclusão de curso com temáticas voltadas a sustentabilidade nos últimos 10 anos, isso gera uma média de 7,7 trabalhos por ano, aproximadamente 8 alunos por ano. Um número bem considerável em relação a média de formação de engenheiros mecânicos por semestre na UFPA, destacando-se a importância de os orientadores empregarem a sustentabilidade em seus trabalhos de conclusão junto com os orientados.

A Tabela 1 ilustra o quantitativo de trabalhos com temas sustentáveis e a relação com o quadro de professores orientadores por área de pesquisa.

Tabela 1 - Número de TCC's em função de professores e áreas de pesquisa.

\begin{tabular}{ccc}
\hline Área de pesquisa & $\begin{array}{c}\text { Trabalhos com temas sobre } \\
\text { sustentabilidade }\end{array}$ & $\begin{array}{c}\text { Número de professores } \\
\text { orientadores }\end{array}$ \\
\hline Térmicas e Fluidos & 31 & 10 \\
Materiais e Processos de Fabricação & 41 & 9 \\
Sistemas mecânicos & 4 & 8 \\
Gestão e Manutenção & 1 & 3 \\
\hline
\end{tabular}

Fonte: Autoria própria (2021). 
O gráfico da Figura 4 ilustra que a área de Materiais e Processo de fabricação detém da maior quantidade de trabalhos que promovem ideias sustentáveis somando 41 trabalhos, $53,25 \%$ do total, seguido de Térmicas e Fluidos com 31 trabalhos $(40,26 \%)$ voltados à temática, logo em seguida vem a área de Sistemas Mecânicos com 4 trabalhos $(5,19 \%)$ e por fim a área de Gestão e Manutenção com 1 trabalho (1,3\%). Isto, de certa forma, evidencia a busca em especial por alternativas e reaproveitamento de materiais frente ao tradicionalmente utilizado, bem como novos processos que gerem menos dispêndio e utilizem de meios mais ecologicamente corretos.

Figura 3 - Quantidade de TCC's defendidos no intervalo de 2010 a 2020 na Faculdade de engenharia Mecânica.

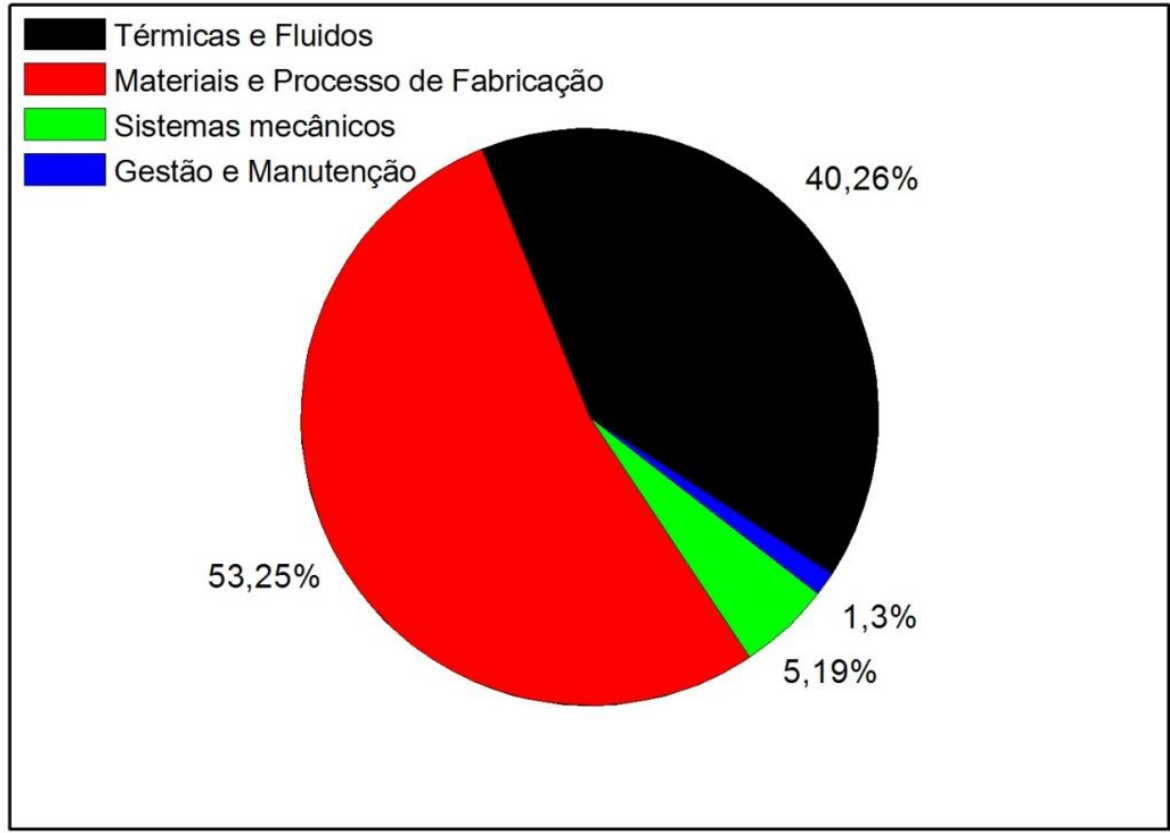

Fonte: Autoria própria (2021).

\subsection{Autorizações/Reconhecimento}

Os autores são responsáveis por garantir o direito de publicar todo o conteúdo de seu trabalho. Se material com direitos autorais foi usado na preparação deste, pode ser necessário obter a devida autorização do detentor dos direitos para a publicação do material em questão. 


\section{CONSIDERAÇÕES FINAIS}

As informações e dados alcançados no presente trabalho foi bem promissor tendo em vista os seguintes destaques:

- O tema sustentabilidade tem sido bem explorado em pesquisas e na geração de Trabalhos de Conclusão de Curso na Faculdade de Engenharia Mecânica.

- Segundo as áreas de pesquisa da Faculdade de Engenharia Mecânica a que teve maior destaque com maior quantidade de TCC'S foi a área de Materiais e Processos de Fabricação.

- Segundo as áreas de pesquisa da Faculdade de Engenharia Mecânica a que teve menor destaque com maior quantidade de TCC'S foi a área de Gestão e Manutenção.

- Foi observado que pesquisas que envolve o tema sustentabilidade está sendo bem explorado pela Faculdade de engenharia Mecânica.

\section{Agradecimentos}

O presente trabalho foi realizado com apoio da Coordenação de Aperfeiçoamento de Pessoal de Nível Superior-Brasil (CAPES)-Código de Financiamento 001. Os autores agradecem também ao CNPq e Pró-reitoria de Ensino de Graduação da Universidade Federal do Pará, Instituto de Tecnologia e à Faculdade de Engenharia Mecânica.

\section{REFERÊNCIAS}

BARTH, M.; RIECKMANN, M. Academic staff development as a catalyst for curriculum change towards education for sustainable development: an output perspective. Journal of Cleaner Production, v. 26, p. 28-36, 2012.

BAZZO, Walter Antonio.; PEREIRA, Luiz Teixeira do Vale.; LINSINGEN, Irlan von. Educação tecnológica: enfoques para o ensino de engenharia. Florianópolis: Editora da UFSC, 2000.

BRITO, W. A. de. Proposta de construção de um currículo integrado de engenharia. In: XXXIX Congresso Brasileiro De Educação Em Engenharia. Blumenau/SC, 03 a 06 de outubro de 2011. Anais.

CARLETTO, M. R. Avaliação de Impacto tecnológico: reflexões, fundamentos e práticas. 1.ed. Curitiba: Ed. UTFPR, 2011.

DWEK, M.; COUTINHO, H.; MATHEUS, F. Por uma formação crítica em engenharia. In: XXXIX Congresso Brasileiro De Educação Em Engenharia. 2011, Blumenau. Anais. Blumenau. Disponível em : https://www.researchgate.net/publication/291802126 Por uma formacao critica em eng enharia. Acesso em 21 abr. 2021.

LINSINGEN, I. V. Engenharia, tecnologia e sociedade: novas perspectivas para uma formação. 2002. Tese (Doutorado). Programa de Pós-Graduação em Educação. Universidade Federal de Santa Catarina, Florianópolis, 2002. Disponível em: http://repositorio.ufsc.br/xmlui/handle/123456789/82388. Acesso em 20 abr. 2021. 
LINSINGEN, I. V.; PEREIRA, L. T. do V.; BAZZO, W. A. O enfoque CTS e a formação em engenharia: convergências curriculares. In: Congresso Brasileiro De Ensino Em Engenharia. 2003, Rio de janeiro. Anais. Rio de janeiro.

LOUREIRO, S. M.; PEREIRA, V. L. D. V.; PACHECO JR, W. A sustentabilidade e o desenvolvimento sustentável na educação em engenharia. Revista Eletrônica em Gestão, Educação e Tecnologia Ambiental. Santa Maria, v. 20 (1), p. 306-324, 2016.

VIEIRA, Cid Willamys Alves; COELHO, Moises Israel Belchior de Andrade. A evolução dos cursos de engenharias na região norte: uma análise sobre os constructos vagas, inscritos, ingressantes. In XXXVII Encontro Nacional de Engenharia de Produção. 2017, Joinville. Anais. Joinville. Disponível em:

http://www.abepro.org.br/biblioteca/TN WIC 247427 33857.pdf. Acesso em 18 de abr. 2021.

WRIGHT, T. S. S.; HORST, N. Exploring the ambiguity: what faculty leaders really think of sustainability in higher education. In: International Journal of Sustainability in Higher Education, v. 14(2), p. 209-227, 2013.

\title{
INVOLVEMENT OF SUSTAINABLE MATERIALS IN TOPICS OF COURSE CONCLUSION WORKS AT THE FACULTY OF MECHANICAL ENGINEERING AT UFPA
}

\begin{abstract}
The use of environmentally friendly materials and socio-environmental sustainability are premises for adapting technology, social well-being to environmental preservation. In view of this, the present work presents, in a quantitative and quantitative way, the use of sustainability in the themes of Course Conclusion Papers (TCC) of the Faculty of Mechanical Engineering of the Federal University of Pará (UFPA) between the years 2010 and 2020 in order to highlight the importance of this theme in the training of engineering professionals. For this, the Lattes curriculum of the professors that make up the faculty of the Faculty of Mechanical Engineering (FEM) at UFPA was used as a database in search of the completed TCC's guidelines. The results show the progress in the preparation of CBT over time as the present article intends. The results also make it clear that the areas of mechanical engineering that provided the greatest amount of Thermal and Fluid TCC's and of Materials and Manufacturing Processes. The results show the progress in the elaboration of TCC over time as the present article intends. The results also make it clear that the areas of mechanical engineering that provided the greatest amount of Thermal and Fluid TCCs and of Materials and Manufacturing Processes.
\end{abstract}

Keywords: Sustainability, Graduation work, Engineering, Education. 